\title{
NONLINEAR PERTURBED INTEGRAL EQUATIONS RELATED TO NONLOCAL BOUNDARY VALUE PROBLEMS
}

\author{
ALBERTO CABADA*, GENNARO INFANTE** AND F. ADRIÁN F. TOJO*** \\ *Departamento de Análise Matemática, Facultade de Matemáticas \\ Universidade de Santiago de Compostela \\ 15782 Santiago de Compostela, Spain \\ E-mail: alberto.cabada@usc.es \\ ** Dipartimento di Matematica e Informatica, Università della Calabria \\ 87036 Arcavacata di Rende, Cosenza, Italy \\ E-mail: gennaro.infante@unical.it \\ ***Departamento de Análise Matemática, Facultade de Matemáticas \\ Universidade de Santiago de Compostela \\ 15782 Santiago de Compostela, Spain \\ E-mail: fernandoadrian.fernandez@usc.es
}

\begin{abstract}
By topological arguments, we prove new results on the existence, non-existence, localization and multiplicity of nontrivial solutions of a class of perturbed nonlinear integral equations. These type of integral equations arise, for example, when dealing with boundary value problems where nonlocal terms occur in the differential equation and/or in the boundary conditions. Some examples are given to illustrate the theoretical results.

Key Words and Phrases: Perturbed integral equation, nonlocal differential equation, nonlinear boundary condition, nontrivial solution, fixed point index, cone.

2010 Mathematics Subject Classification: 45G10, 34A34, 34B10, 34B15, 34B18, 34K10.

Acknowledgements. A. Cabada and F. A. F. Tojo were partially supported by Ministerio de Educación y Ciencia, Spain, and FEDER, Project MTM201343014-P. F.A.F. Tojo was partially supported by Xunta de Galicia (Spain), project EM2014/032; FPU scholarship, Ministerio de Educación, Cultura y Deporte, Spain and a Fundación Barrie Scholarship. G. Infante was partially supported by G.N.A.M.P.A. - INdAM (Italy). This paper was mostly written during a visit of F.A.F. Tojo to the Dipartimento di Matematica e Informatica of the Università della Calabria. F.A.F. Tojo is grateful to the people of the aforementioned Dipartimento for their kind and warm hospitality. The authors wish to acknowledge their gratitude to Dr Filomena Cianciaruso and Professor Paolamaria Pietramala, who helped improve this paper with their fruitful suggestions and comments.
\end{abstract}




\section{REFERENCES}

[1] E. Alves, T.F. Ma, M.L. Pelicer, Monotone positive solutions for a fourth order equation with nonlinear boundary conditions, Nonlinear Anal., 71(2009), 3834-3841.

[2] H. Amann, Fixed point equations and nonlinear eigenvalue problems in ordered Banach spaces, SIAM. Rev., 18(1976), 620-709.

[3] D. Andrade, T.F. Ma, Numerical solutions for a nonlocal equation with reflection of the argument, Neural Parallel Sci. Comput., 10(2002), 227-233.

[4] D. Bugajewski, M. Zima, On the spectral radius of linearly bounded operators and existence results for functional-differential equations, Proc. Fourth International Conf. on Dynamical Systems and Differential Equations, (2002), 147-155.

[5] A. Cabada, An overview of the lower and upper solutions method with nonlinear boundary value conditions, Bound. Value Probl. (2011), Art. ID 893753, 18 pp.

[6] A. Cabada, G. Infante, F.A.F. Tojo, Nontrivial solutions of perturbed Hammerstein integral equations with reflections, Bound. Value Probl., 86(2013).

[7] A. Cabada, G. Infante, F.A.F. Tojo, Nonzero solutions of perturbed Hammerstein integral equations with deviated arguments and applications, Topol. Methods Nonlinear Anal., to appear.

[8] M. Chipot, J.F. Rodrigues, On a class of nonlinear nonlocal elliptic problems, Math. Model. Num. Anal., 26(1992), 447-467.

[9] R. Conti, Recent trends in the theory of boundary value problems for ordinary differential equations, Boll. Un. Mat. Ital., 22(1967), 135-178.

[10] K. Deimling, Nonlinear Functional Analysis, Springer-Verlag, Berlin, 1985. Reprinted, Dover Publications.

[11] V. Dolezal, Some results on the invertibility of nonlinear operators, Circuits Systems Signal Process, 17-6(1998), 683-690.

[12] V. Dolezal, The invertibility of operators and contraction mappings, Circuits Systems Signal Process, 18-2(1999), 183-187.

[13] H. Fan, R. Ma, Loss of positivity in a nonlinear second order ordinary differential equations, Nonlinear Anal., 71(2009), 437-444.

[14] R. Figueroa, R.L. Pouso, Minimal and maximal solutions to second-order boundary value problems with state-dependent deviating arguments, Bull. London Math. Soc., 43(2011), 164-174.

[15] D. Franco, D. O'Regan, J. Perán, Fourth-order problems with nonlinear boundary conditions, J. Comput. Appl. Math., 174(2005), 315-327.

[16] D. Franco, G. Infante, D. O'Regan, Positive and nontrivial solutions for the Urysohn integral equation, Acta Math. Sinica, 22(2006), 1745-1750.

[17] C.S. Goodrich, On nonlocal BVPs with nonlinear boundary conditions with asymptotically sublinear or superlinear growth, Math. Nachr., 285(2012), 1404-1421.

[18] C.S. Goodrich, Positive solutions to boundary value problems with nonlinear boundary conditions, Nonlinear Anal., 75(2012), 417-432.

[19] C.S. Goodrich, On nonlinear boundary conditions satisfying certain asymptotic behavior, Nonlinear Anal., 76(2013), 58-67.

[20] C.S. Goodrich, A note on semipositone boundary value problems with nonlocal, nonlinear boundary conditions, Arch. Math. (Basel), 103(2014), 177-187.

[21] C.S. Goodrich, Semipositone boundary value problems with nonlocal, nonlinear boundary conditions, Adv. Differential Equations, 20(2015), 117-142.

[22] P. Guidotti, S. Merino, Gradual loss of positivity and hidden invariant cones in a scalar heat equation, Differential Integral Equations, 13(2000), 1551-1568.

[23] D. Guo, V. Lakshmikantham, Nonlinear Problems in Abstract Cones, Academic Press, Boston, 1988.

[24] G. Infante, Eigenvalues of some non-local boundary-value problems, Proc. Edinb. Math. Soc., 46(2003), $75-86$.

[25] G. Infante, Nonzero solutions of second order problems subject to nonlinear BCs, Dynamic systems and applications, Vol. 5, 222-226, Dynamic, Atlanta, GA, 2008. 
[26] G. Infante, Nonlocal boundary value problems with two nonlinear boundary conditions, Commun. Appl. Anal., 12(2008), 279-288.

[27] G. Infante, Positive solutions of a doubly nonlocal boundary value problem, Commun. Appl. Anal., 18(2014), 147-154.

[28] G. Infante, P. Pietramala, Perturbed Hammerstein integral inclusions with solutions that change sign, Comment. Math. Univ. Carolin., 50(2009), 591-605.

[29] G. Infante, P. Pietramala, A cantilever equation with nonlinear boundary conditions Electron. J. Qual. Theory Differ. Equ., Spec. Ed. I, 15(2009), 1-14.

[30] G. Infante, P. Pietramala, F.A.F. Tojo, Nontrivial solutions of local and nonlocal Neumann boundary value problems, Proc. Roy. Soc. Edinburgh Sect. A, to appear.

[31] G. Infante, J.R.L. Webb, Nonzero solutions of Hammerstein integral equations with discontinuous kernels, J. Math. Anal. Appl., 272(2002), 30-42.

[32] G. Infante, J.R.L. Webb, Three point boundary value problems with solutions that change sign, J. Integral Equations Appl., 15(2003), 37-57.

[33] G. Infante, J.R.L. Webb, Nonlinear nonlocal boundary value problems and perturbed Hammerstein integral equations, Proc. Edinb. Math. Soc., 49(2006), 637-656.

[34] T. Jankowski, Solvability of three point boundary value problems for second order differential equations with deviating arguments, J. Math. Anal. Appl., 312(2005), 620-636.

[35] T. Jankowski, Multiple solutions for a class of boundary-value problems with deviating arguments and integral boundary conditions, Dynam. Systems Appl., 19(2010), 179-188.

[36] T. Jankowski, Positive solutions to second-order differential equations with dependence on the first-order derivative and nonlocal boundary conditions, Bound. Value Probl., 8(2013).

[37] G.L. Karakostas, Existence of solutions for an $n$-dimensional operator equation and applications to BVPs, Electron. J. Differential Equations, (2014), no. 71, 17 pp.

[38] G.L. Karakostas, P. Ch. Tsamatos, Existence of multiple positive solutions for a nonlocal boundary value problem, Topol. Meth. Nonlinear Anal., 19(2002), 109-121.

[39] G.L. Karakostas, P. Ch. Tsamatos, Multiple positive solutions of some Fredholm integral equations arisen from nonlocal boundary-value problems, Electron. J. Differential Equations, (2002), no. 30,17 pp.

[40] M.A. Krasnosel'skiı̌, P.P. Zabreı̌ko, Geometrical Methods of Nonlinear Analysis, SpringerVerlag, Berlin, 1984.

[41] K.Q. Lan, Multiple positive solutions of Hammerstein integral equations with singularities, Differential Equations and Dynamical Systems, 8(2000), 175-195.

[42] R. Ma, A survey on nonlocal boundary value problems, Appl. Math. E-Notes, 7(2001), 257-279.

[43] J.J. Nieto, J. Pimentel, Positive solutions of a fractional thermostat model, Bound. Value Probl., 2013:5(2013).

[44] S.K. Ntouyas, Nonlocal initial and boundary value problems: a survey, Handbook of Differential Equations: Ordinary Differential Equations, Vol. II, 461-557, Elsevier B. V., Amsterdam, 2005.

[45] D. Piao, Pseudo almost periodic solutions for differential equations involving reflection of the argument, J. Korean Math. Soc., 41(2004), 747-754.

[46] D. Piao, Periodic and almost periodic solutions for differential equations with reflection of the argument, Nonlinear Anal., 57(2004), 633-637.

[47] D. Piao, Na Xin, Bounded and almost periodic solutions for second order differential equation involving reflection of the argument, arXiv:1302.0616.

[48] M. Picone, Su un problema al contorno nelle equazioni differenziali lineari ordinarie del secondo ordine, Ann. Scuola Norm. Sup. Pisa Cl. Sci., 10(1908), 1-95.

[49] P. Pietramala, A note on a beam equation with nonlinear boundary conditions, Bound. Value Probl., (2011), Art. ID 376782, 14 pp.

[50] A. Štikonas, A survey on stationary problems, Green's functions and spectrum of SturmLiouville problem with nonlocal boundary conditions, Nonlinear Anal. Model. Control, 19(2014), 301-334.

[51] W. Szatanik, Quasi-solutions for generalized second order differential equations with deviating arguments, J. Comput. Appl. Math., 216(2008), 425-434. 
[52] W. Szatanik, Minimal and maximal solutions for integral boundary value problems for the second order differential equations with deviating arguments, Dynam. Systems Appl., 19(2010), $87-96$.

[53] J.R.L. Webb, Solutions of nonlinear equations in cones and positive linear operators, J. London Math. Soc., 82(2010), 420-436.

[54] J.R.L. Webb, A class of positive linear operators and applications to nonlinear boundary value problems, Topol. Meth. Nonlinear Anal., 39(2012), 221-242.

[55] J.R.L. Webb, Existence of positive solutions for a thermostat model, Nonlinear Anal. Real World Appl., 13(2012), 923-938.

[56] J.R.L. Webb, G. Infante, Positive solutions of nonlocal boundary value problems: a unified approach, J. London Math. Soc., 74(2006), 673-693.

[57] J.R.L. Webb, G. Infante, Positive solutions of nonlocal boundary value problems involving integral conditions, NoDEA - Nonlinear Differential Equations Appl., 15(2008), 45-67.

[58] J.R.L. Webb, G. Infante, Nonlocal boundary value problems of arbitrary order, J. London Math. Soc., 79(2009), 238-258

[59] J.R.L. Webb, K.Q. Lan, Eigenvalue criteria for existence of multiple positive solutions of nonlinear boundary value problems of local and nonlocal type, Topol. Meth. Nonlinear Anal., 27(2006), $91-115$.

[60] W.M. Whyburn, Differential equations with general boundary conditions, Bull. Amer. Math. Soc., 48(1942), 692-704.

[61] J. Wiener, A.R. Aftabizadeh, Boundary value problems for differential equations with reflection of the argument, Internat. J. Math. Math. Sci., 8(1985), 151-163.

[62] Z. Yang, Positive solutions to a system of second-order nonlocal boundary value problems, Nonlinear Anal., 62(2005), 1251-1265.

[63] Z. Yang, Positive solutions of a second-order integral boundary value problem, J. Math. Anal. Appl., 321(2006), 751-765.

[64] M. Zima, Applications of the spectral radius to some integral equations, Comment. Math. Univ. Carolin., 36(1995), 695-703.

Received: January 27, 2016; Accepted: April 18, 2016. 